\title{
"Primum non nocere": the role of hydroxyethyl starch 130/0.4 in cerebral resuscitation
}

\author{
John M. Murkin, MD
}

Received: 10 September 2012/ Accepted: 20 September 2012/Published online: 28 November 2012

(C) Canadian Anesthesiologists' Society 2012

\begin{abstract}
Editor's note
A recent editorial (Miller DR. Update to readers and authors on ethical and scientific misconduct: retraction of the "Boldt articles" - Can J Anesth 2011: 58: 777-81) addressed the retraction of articles authored by Dr. Joachim Boldt et al. A number of the Boldt articles reported on hydroxyethyl starches (HES), including trials evaluating HES 130/0.4. As a result of the article retractions in this journal and others, there is ongoing need to determine more conclusively the role of these intravenous solutions in clinical practice. A particularly controversial topic is the role of hydroxyethyl starches in traumatic brain injury. In light of the controversy in the literature and findings from recent studies, we offer the following invited editorial perspective by Dr. John Murkin, Professor of Anesthesiology from the Schulich School of Medicine, University of Western Ontario, London, Ontario.
\end{abstract}

Donald R. Miller, M.D., Editor-in-Chief

The role of various fluid management strategies as primary therapeutic modalities for cerebral resuscitation in the amelioration of ischemic, traumatic, or post-hemorrhagic brain injury has been investigated for decades. From the initial studies of hypervolemic hemodilution $(\mathrm{HH})$ in the early 1980s and onwards, various fluid therapies targeting hemorheology and utilizing osmotherapy and hypernatremic therapies have shown varying degrees of promise in a

J. M. Murkin, MD ( $₫)$

Department of Anesthesiology and Perioperative Medicine,

Schulich School of Medicine, University of Western Ontario,

London, ON, Canada

e-mail: john.murkin@lhsc.on.ca variety of experimental and clinical settings, ${ }^{1,2}$ though controversy regarding overall efficacy remains. ${ }^{3}$ Given the all too recent retractions of numerous fraudulent publications and the subsequent discrediting of a large body of literature dealing with the safety and efficacy of hydroxyethyl starch (HES) 130/0.4, fundamental concerns have been raised. This unease necessitates a critical reappraisal of the role of HES 130/0.4 in general ${ }^{4}$ and, in particular, a reevaluation of its therapeutic administration to those patients with acute central nervous system injury. ${ }^{5}$ Given the complexities of the subject area and the heterogeneity of related clinical trials, the purpose of this editorial is to provide a clinical perspective on the more recent studies related to the role of HES in cerebral resuscitation.

In concert with the developments in HH therapy, ongoing studies have confirmed the primacy of inflammatory processes in the pathogenesis of neuronal injury and cell death. ${ }^{6}$ At the same time, the identification of a family of aquaporin water channels as key components in cerebral tissue water regulation (specifically the role of glial water channel aquaporin-4 [AQP4] as etiologic in the development of both cytotoxic edema and the reabsorption of extracellular edema fluid and the development of vasogenic edema ${ }^{7}$ - and the impact of hypernatremia and hypoosmolar therapy on AQP4 bioavailability) ${ }^{8}$ has re-emphasized the role of basal sodium and fluid management in the care of patients with acute brain injury ${ }^{9}$ while concomitantly providing putative mechanisms for some of the salutary effects of HES observed in animal models of cerebral ischemia. ${ }^{10}$

As such, preliminary studies have indicated a beneficial effect of HES in decreasing blood brain barrier permeability and edema formation in spinal cord ischemia ${ }^{11}$ and have also revealed the further expression of a cerebral vascular anti-inflammatory effect with significant inhibition of leukocyte adhesion and vascular permeability in a global hypoxia 
model. ${ }^{12}$ These results have given rise to several phase II preclinical studies investigating the safety of newer generation HES in patients with ischemic stroke. ${ }^{13,14}$ Subsequent animal studies have also shown a degree of cerebral protection manifested as decreased infarct volumes in response to focal ischemic injury. Then again, any potential direct clinical relevance was minimized inasmuch as this model employed pretreatment with HES 130/0.4 to a hematocrit of 25-30\% prior to development of $120 \mathrm{~min}$ of focal ischemia. ${ }^{15}$

As initially conceived, hydroxyethyl starches were developed from amylopectin, a highly branched starch compound rapidly hydrolyzed by plasma amylases, for use by the military seeking a low-mass high-volume expansion fluid as compensation for acute blood loss. ${ }^{14}$ Subsequently, different manufacturing processes utilizing either corn or potato starches have resulted in a variety of different HES solutions of varying in vitro molecular weight (GMW) and degrees of molar substitution, i.e., a measure of the ratio of substitution of hydroxyethyl groups for glucose units, as well as differing substitution types, identified as the $\mathrm{C} 2 / \mathrm{C} 6$ hydroxyethylation ratio. Both higher degrees of substitution and larger C2/C6 ratios result in slower hydrolysis and clearance and a more prolonged intravascular osmotic effect. ${ }^{16}$ Initially, HES with a high GMW (480 kD) and a high molar substitution (0.7) was developed (6\% Hespan ${ }^{\circledR}$, B Braun, Irvine, CA, USA), but these large molecules subsequently proved difficult to eliminate and gave rise to a variety of coagulation and tissue storage complications when administered in higher dosages (>20 mL $\cdot \mathrm{kg}^{-1} / 24 \mathrm{hr}$ ). In response, HES with a GMW of 200 $\mathrm{kD}$ and a lower molar substitution of 0.5 was developed (10\% Pentaspan ${ }^{2}$, Bristol-Myers Squibb, Montreal, QC, Canada), allowing much quicker renal elimination, although a degree of platelet dysfunction and increased risk of bleeding has still been shown. ${ }^{17,18}$

Subsequent development of HES with a GMW of $130 \mathrm{kD}$ and a molar substitution of 0.4 was undertaken, but molecules with low molar substitution are rapidly cleaved enzymatically by amylase, resulting in a short intravascular volume effect. ${ }^{16}$ Since the metabolism of HES also depends on the $\mathrm{C} 2 / \mathrm{C} 6$ hydroxyethylation ratio, wherein the higher the ratio the slower the breakdown, HES 130/0.4 with a higher $\mathrm{C} 2 / \mathrm{C} 6$ ratio $(>8)$ was developed (Voluven ${ }^{\circledR}$ $6 \%$, Fresenius Kabi Deutschland $\mathrm{GmbH}$, Bad Homburg, Germany), giving rise to a synthetic molecule with a longer intravascular presence but producing a significant decrease in tissue persistence. As confirmation, in a long-term animal model using radiolabelled ${ }^{14} \mathrm{C}$-HES $130 / 0.4$, a residual of $0.6 \%$ of the administered dose was found at day 52 compared with a residual of $2.45 \%$ of the administered dose of ${ }^{14} \mathrm{C}$-HES $200 / 0.5 .{ }^{19}$ This represents the current generation of HES 130/0.4, which has been available for the past several years in North America as Voluven ${ }^{\circledR}$ and, until recently, has seen ever-widening clinical application.
Unfortunately, there is a lack of relevant published clinical data in cerebral resuscitation patients apart from the two studies mentioned above, one which involved 106 acute stroke patients who received titrated hypervolemic $\mathrm{HH}$ with either HES $130 / 0.4$ or $0.9 \% \mathrm{NaCl}$ as a phase II safety study, ${ }^{13}$ and the other which involved 40 acute stroke patients who were similarly randomized but received continuous fluid therapy totaling $6.5 \mathrm{~L}$ over four days. ${ }^{14}$ Superior therapeutic efficacy was not shown in either study. Cumulatively, the therapeutic safety equivalence claimed in both of these studies is based on the documented administration of HES 130/0.4 in a relatively small number $(n<75)$ of acute stroke patients. ${ }^{13,14}$ In a further study, 31 patients with severe head injury were randomized to treatment over multiple days with repeated large doses of HES 130/0.4 [cumulatively 19 (16) L] or HES 200/0.5 and albumin [cumulatively 22 (11) L]. The conclusion was that HES $130 / 0.4$ can be used safely in repeated dosages of up to $70 \mathrm{~mL} \cdot \mathrm{kg}^{-1} \cdot \mathrm{day}^{-1}$ over several days in critically ill head trauma patients. ${ }^{20}$ Nevertheless, very serious concerns have been raised regarding the relative lack of power in all three of these studies to assess safety related to potential bleeding or other complications adequately. ${ }^{21,22}$

A further degree of caution surrounding administration of HES 130/0.4 is now prudent given recent findings. For example, in a prospective randomized study of 798 patients with severe sepsis increased risk of death and requirement for renal replacement therapy was shown in patients who were resuscitated with HES 130/0.4 vs Ringer's acetate, ${ }^{23}$ a finding not inconsistent with the results of another recent prospective sequential therapy review of colloids $v s$ crystalloids also in a large group of patients with severe sepsis in which administration of HES and gelatin were independent risk factors for acute kidney injury. ${ }^{24}$ The just published results of the Crystalloid versus Hydroxyethyl Starch Trial (CHEST) provide much needed objective data of sufficient power to rigorously determine issues of safety and therapeutic efficacy. ${ }^{25}$ This trial involved 7000 critically ill patients randomized to receive either 6\% HES $130 / 0.4$ or $0.9 \%$ sodium chloride for all fluid resuscitation requirements and showed no significant difference between groups with respect to the primary outcome of all-cause mortality at 90 days "[597 of 3315 patients (18.0\%) in the HES group and 566 of $3336(17.0 \%)$ in the saline group (relative risk in the HES group, 1.06; 95\% confidence interval, 0.96 to $1.18 ; P=0.26$ ]", while HES $130 / 0.4$ was associated with significantly more adverse events $(5.3 \%$ vs. $2.8 \%, P<0.001)$, renal injury $(34.6 \%$ and $38.0 \%$ of patients, respectively $(P=0.005)$ and requirement for renal-replacement therapy (relative risk, $1.21 ; 95 \% \mathrm{CI}$, 1.00 to $1.45 ; P=0.04){ }^{25}$

It remains to be determined whether these adverse outcomes are in any way related to the very small but 
surprisingly persistent tissue presence of detectable residua of HES biometabolites days and weeks after administration. ${ }^{19}$ It is instructive, however, that earlier concerns had been raised regarding the potential for complications related to renal impairment, coagulopathy, and delayed refractory pruritus associated with HES 130/0.4. ${ }^{26}$

In conclusion, severe sepsis represents a very specific and high-risk group of patients with multiple comorbidities and numerous potential confounding factors; however, this association of significantly heightened risk in the absence of compelling evidence of therapeutic efficacy argues for reconsideration of the use of HES 130/0.4 in patients with acute brain injury in the absence of more rigorous substantiation of relative safety. ${ }^{5}$ Even further, these results should also caution against an implicit assumption of safety and the otherwise routine administration of HES 130/0.4 and related compounds in a variety of other unproven but clinically common situations. ${ }^{27}$

\section{«Primum non nocere »: La place de l'hydroxyéthylamidon130/ 0,4 en réanimation cérébrale}

\section{Note du rédacteur en chef}

Un éditorial récent (Miller DR. Mise à jour destinée aux lecteurs et aux auteurs concernant la malhonnêteté scientifique et les infractions aux règles d'éthique : la rétractation des articles du Dr Boldt Can J Anesth 2011: 58: 777-81) abordait la rétractation des articles soumis par le Dr Joachim Boldt et coll. Un certain nombre d'articles de Boldt et coll. traitaient de l'hydroxyéthylamidon (HES), et ceci comprenait des études évaluant le HES 130/0,4. Suite à la rétractation des articles dans ce journal et dans d'autres, il est maintenant nécessaire de tirer des conclusions plus claires sur le rôle de ces solutions intraveineuses en pratique clinique. Un thème particulièrement controversé est la place de l'hydroxyéthylamidon dans les lésions cérébrales traumatiques. À la lumière de cette controverse dans la littérature et des constatations tirées d'études récentes, nous vous proposons le point de vue de notre éditorialiste invité, le Dr John Murkin, professeur d'anesthésiologie à la Schulich School of Medicine, University of Western Ontario, London, Ontario.

Donald R. Miller, M.D., Rédacteur en chef

L'intérêt des différentes stratégies de prise en charge des liquides comme modalité thérapeutique primaire de la réanimation en vue de l'amélioration d'une lésion cérébrale ischémique, traumatique ou post hémorragique a été étudié depuis des décennies. Depuis les premières études sur l'hémodilution hypervolémique $(\mathrm{HH})$ au début des années 1980, puis au cours des années suivantes, diverses thérapies impliquant des liquides ciblant l'hémorhéologie et utilisant des propriétés thérapeutiques d'osmothérapie et d'hypernatrémie ont été prometteuses à divers degrés dans un grand nombre de contextes expérimentaux et cliniques, ${ }^{1,2}$ bien que la controverse entourant leur efficacité globale persiste. ${ }^{3}$ Compte tenu des rétractations très récentes de nombreuses publications frauduleuses et du discrédit jeté sur un vaste ensemble de la littérature traitant de l'efficacité et de l'innocuité de l'hydroxyéthylamidon (HES) 130/0,4, des questions fondamentales ont été soulevées. Ce malaise nécessite une réévaluation critique du rôle du HES 130/0,4 en général ${ }^{4}$ et une réévaluation de son administration à visée thérapeutique chez des patients ayant une lésion aiguë du système nerveux central, en particulier. ${ }^{5}$ Considérant la complexité de ce domaine et l'hétérogénéité des essais cliniques qui s'y rapportent, l'objectif de cet éditorial est de donner un point de vue clinique sur les études les plus récentes concernant le rôle du HES dans la réanimation cérébrale.

Parallèlement au développement du traitement $\mathrm{HH}$, les études en cours ont confirmé le caractère prédominant des processus inflammatoires dans la pathogenèse des lésions neuronales et de la mort cellulaire. ${ }^{6}$ En même temps, l'identification d'une famille de canaux d'aquaporine (ou canaux hydriques) comme principal composant de la régulation de l'eau dans le tissu cérébral (plus particulièrement le rôle du canal d'eau aquaporine-4 [AQP4] des cellules gliales comme point de départ étiologique de l'œdème cytotoxique, de la réabsorption du liquide d'œdème extracellulaire et de la progression de l'œdème vasogénique, ${ }^{7}$ et l'impact de l'hyponatrémie et du traitement hypo-osmolaire sur la biodisponibilité des $\mathrm{AQP} 4)^{8}$ a remis en lumière le rôle de la natrémie de départ et de la gestion des liquides dans les soins de patients ayant des lésions cérébrales aiguës. ${ }^{9}$ Cela a également ouvert la voie à des modes d'action possibles pour certains des effets bénéfiques du HES, observés dans des modèles animaux d'ischémie cérébrale. ${ }^{10}$

Parmi ceux-ci, les études préliminaires ont montré un effet bénéfique du HES sur la baisse de perméabilité de la barrière hémato-encéphalique et l'apparition d'un œdème dans la moelle épinière ischémique. ${ }^{11}$ Ces études ont également révélé l'expression d'un effet anti-inflammatoire cérébrovasculaire supplémentaire avec une inhibition significative de l'adhésion leucocytaire et de la perméabilité vasculaire dans un modèle d'hypoxie globale. ${ }^{12}$ Ces résultats ont débouché sur plusieurs études précliniques de Phase II analysant l'innocuité d'une nouvelle génération de HES chez des patients ayant présenté un accident vasculaire cérébral (AVC) ischémique. ${ }^{13,14}$ Des études ultérieures chez l'animal ont également montré un certain degré de protection cérébrale se traduisant par une diminution du volume de l'infarctus 
produit par une lésion ischémique focale. Ici encore, toute pertinence clinique directe d'un effet potentiel a été minimisée dans la mesure où un prétraitement au HES 130/0,4 abaissant l'hématocrite à une valeur de $25 \%$ à $30 \%$ avait été appliqué avant une ischémie localisée d'une durée de $120 \mathrm{~min}^{15}$

Selon sa conception initiale, l'hydroxyéthylamidon a été développé à partir de l'amylopectine, un amidon hautement ramifié et rapidement hydrolysé par les amylases plasmatiques que les militaires - cherchant un liquide permettant une forte expansion volumique pour une faible masse - devaient pouvoir utiliser pour compenser une perte de sang aiguë. ${ }^{14}$ Ultérieurement, différents procédés de fabrication faisant appel aux amidons de maïs ou de pomme de terre ont débouché sur la mise au point de plusieurs solutions de HES avec un poids moléculaire (PM) in vitro variable et différents degrés de substitution molaire, qui est définie comme la mesure du rapport de substitution d'unités de glucose par des groupes hydroxyéthyles, ainsi que différents types de substitution (identifié comme étant le rapport d'hydroxyéthylation $\mathrm{C} 2 / \mathrm{C} 6$ ). Des degrés de substitution et des rapports C2/C6 plus élevés ont abouti à un ralentissement de l'hydrolyse et de la clairance et à un allongement de la durée de l'effet osmotique intravasculaire. ${ }^{16}$ À l'origine, un HES avec un PM élevé $(480 \mathrm{kD})$ et un haut taux de substitution molaire $(0,7)$ a été développé (6\% Hespan ${ }^{\circledR}$, B Braun, Irvine, CA, USA), mais ces grosses molécules se sont avérées ultérieurement difficiles à éliminer et ont donné lieu à un certain nombre de complications sur la coagulation et la distribution tissulaire quand elles étaient administrées à des doses élevées (> $20 \mathrm{~mL} \cdot \mathrm{kg}^{-1} / 24 \mathrm{~h}$ ). Aussi, un HES ayant un PM de $200 \mathrm{kD}$ et un taux de substitution molaire de 0,5 a été développé (10\% Pentaspan ${ }^{\circledR}$, Bristol-Myers Squibb, Montréal, QC, Canada) permettant une élimination rénale beaucoup plus rapide, même si un certain niveau de dysfonction plaquettaire et un risque hémorragique accru était encore constaté. ${ }^{17,18}$

Le développement ultérieur d'un HES ayant un PM de $130 \mathrm{kD}$ et un taux de substitution molaire de 0,4 a été entrepris, mais les molécules ayant un faible taux de substitution molaire ont été rapidement scindées par l'amylase, n'entraînant qu'un effet de volume intravasculaire de courte durée. ${ }^{16}$ Considérant que le métabolisme du HES dépend également du rapport d'hydroxyéthylation $\mathrm{C} 2 / \mathrm{C} 6$ (plus le rapport est élevé, plus la dégradation de la molécule est lente), un HES 130/0,4 ayant un rapport C2/C6 plus élevé $(>8)$ a été développé (Voluven $\AA$ 6\%, Fresenius Kabi Deutschland $\mathrm{GmbH}$, Bad Homburg, Allemagne); cela a donné naissance à une molécule synthétique présente plus longtemps dans le compartiment intravasculaire, mais persistant significativement moins longtemps dans les tissus. Pour le confirmer, une étude à long terme chez l'animal utilisant du HES marqué au ${ }^{14} \mathrm{C}\left({ }^{14} \mathrm{C}\right.$-HES $\left.130 / 0,4\right)$ a trouvé une dose résiduelle de $0,6 \%$ de la dose administrée au bout de 52 jours, comparativement à un résidu de 2,45\% d'une dose $\mathrm{de}^{14} \mathrm{C}$-HES $200 / 0,5 .{ }^{19}$ Cela représente la génération actuelle de HES 130/0,4, qui est disponible depuis quelques années en Amérique du Nord sous le nom de Voluven® et dont les applications cliniques se sont élargies, jusqu'à maintenant.

Malheureusement, nous manquons de données cliniques pertinentes pour les patients de réanimation cérébrale, en dehors des deux études citées plus haut, l'une ayant inclus 106 patients souffrant d'AVC aigu ayant reçu un traitement hypervolémique $\mathrm{HH}$ titré par du HES 130/0,4 ou duNaCl $0,9 \%$ dans le cadre d'une étude d'innocuité de Phase II ${ }^{13} \mathrm{et}$ l'autre ayant inclus 40 patients souffrant d'AVC aigu qui avaient été randomisés de manière identique, mais avaient continué à recevoir une quantité totale de $6,5 \mathrm{~L}$ de liquides en quatre jours. ${ }^{14}$ Aucune supériorité thérapeutique n'a été constatée dans ces deux études. Prises ensemble, l'équivalence d'innocuité thérapeutique affirmée dans ces deux études repose sur l'administration documentée de HES 130/0,4 chez un nombre relativement faible de patients $(n<75)$ victimes d'un AVC. ${ }^{13,14}$ Dans une autre étude, 31 patients ayant une lésion intracrânienne grave ont été randomisés pour recevoir un traitement de plusieurs jours avec de grandes doses répétées de HES 130/0,4 [19 (16) L cumulés] ou de HES 200/0,5 et d'albumine [22 (11) L cumulés]. La conclusion de cette dernière étude était que le HES 130/0,4 pouvait être utilisé sans danger à des doses répétées pouvant atteindre $70 \mathrm{~mL} \cdot \mathrm{kg}^{-1} \cdot$ jour $^{-1}$ pendant plusieurs jours chez des patients ayant untraumatisme intracrânien grave. $^{20}$ Néanmoins, des préoccupations très sérieuses ont été soulevées devant le manque de puissance de ces trois études pour l'évaluation adéquate de l'innocuité en rapport avec un risque d'hémorragie ou d'autres complications. $^{21,22}$

Il est maintenant prudent de faire une mise en garde sur l'administration de HES 130/0,4, compte tenu de constatations récentes. Par exemple, dans une étude randomisée prospective ayant inclus 798 patients ayant un sepsis grave, une augmentation du risque de décès et d'un traitement de remplacement rénal est apparue chez les patients qui avaient été réanimés à l'aide de HES 130/0,4 comparativement à du soluté d'acétate Ringer, ${ }^{23}$ une constatation qui ne va pas à l'encontre d'une autre analyse prospective récente du traitement séquentiel aux colloïdes ou aux cristalloïdes, chez un grand nombre de patients ayant un sepsis sévère: l'administration de HES ou de gélatine a constitué dans ce cas des facteurs de risque indépendants de lésion rénale aiguë. ${ }^{24}$ Les résultats fraîchement sortis de presse de l'étude CHEST (Crystalloid versus HydroxyethylStarch Trial) fournissent des données objectives très attendues, avec une puissance suffisante, pour éclairer de façon rigoureuse les questions d'innocuité 
et d'efficacité thérapeutique. ${ }^{25}$ Cette étude a porté sur 7000 patients gravement malades, randomisés pour recevoir du HES 130/04 à $6 \%$ ou du chlorure de sodium à $0,9 \%$ pour répondre à tous leurs besoins de réanimation et n'a démontré aucune différence significative entre les groupes en ce qui touche au critère d'évaluation principal de mortalité toutes causes confondues à 90 jours, « [597 des 3315 patients $(18,0 \%)$ dans le groupe HES et 566 des 3336 $(17,0 \%)$ des patients du groupe chlorure de sodium (risque relatif dans le groupe HES, 1,06; intervalle de confiance $95 \%, 0,96$ à 1,$18 ; P=0,26]$ ], alors que le HES 130/0,4 a été associé à un nombre significativement plus élevé d'effets néfastes $(5,3 \%$ vs. $2,8 \%, P<0,001)$, de lésions rénales (34,6\% et $38,0 \%$ de patients, respectivement $(P=0,005)$ et le besoin de traitement de remplacement rénal (risque relatif, 1,21 ; IC $95 \%, 1,00$ à 1,$45 ; P=0,04) .^{25}$

Il reste à déterminer si ces évolutions funestes sont à rapprocher d'une façon quelconque de la présence dans les tissus d'une quantité très faible - mais étonnamment persistante - de résidu détectable de métabolites du HES, des jours ou des semaines après son administration. ${ }^{19}$ Il est instructif de noter, toutefois, que des préoccupations antérieures avaient été soulevées à propos du risque de complications liées à une insuffisance rénale, une coagulopathie ou à un prurit réfractaire tardif, associé au HES 130/0,4. ${ }^{26}$

En conclusion, les patients ayant un sepsis sévère constituent un groupe très spécifique et à haut risque, présentant de multiples comorbidités et de nombreux facteurs confondants potentiels.Toutefois, l'augmentation significative de ce risque associée à l'absence de données probantes irréfutables de l'efficacité thérapeutique plaident en faveur d'un réexamen de l'utilisation du HES 130/0,4 chez des patients victimes de lésions cérébrales aiguës, en l'absence de bases plus rigoureuses de son innocuité relative. ${ }^{5}$ De plus, ces résultats doivent aussi inviter à la prudence et conduire à rejeterl'innocuité implicite supposée du HES 130/0,4 et des produits apparentés, au cours d'une administration de routine, dans de nombreuses situations cliniques en l'absence de preuves. ${ }^{27}$

Competing interests None declared.

\section{References}

1. Wood JH, Kee DB Jr. Hemorheology of the cerebral circulation in stroke. Stroke 1985; 16: 765-72.

2. Kelley RE, Martin-Schild S. Ischemic stroke: emergencies and management. Neurol Clin 2012; 30: 187-210.

3. Asplund $K$. Haemodilution for acute ischaemic stroke. Cochrane Database Syst Rev 2002; 4: CD000103.

4. Hartog CS, Bauer M, Reinhart $K$. The efficacy and safety of colloid resuscitation in the critically ill. Anesth Analg 2011; 112: 156-64.
5. Reinhart K, Perner A, Sprung CL, et al. Unless high-quality clinical data show they are safe, synthetic colloids should not be used in patients with head injury. Intensive Care Med 2012; 38: 1563-4.

6. Lambertsen $K L$, Biber $K$, Finsen B. Inflammatory cytokines in experimental and human stroke. J Cereb Blood Flow Metab 2012; doi:10.1038/jcbfm.2012.88.

7. Zador Z, Stiver S, Wang V, Manley GT. Role of aquaporin-4 in cerebral edema and stroke. Handb Exp Pharmacol 2009; 190: 159-70.

8. Zeynalov E, Chen CH, Froehner SC, et al. The perivascular pool of aquaporin-4 mediates the effect of osmotherapy in postischemic cerebral edema. Crit Care Med 2008; 36: 2634-40.

9. Wright WL. Sodium and fluid management in acute brain injury. Curr Neurol Neurosci Rep 2012; 12: 466-73.

10. Zivin JA. Hydroxyethyl starch reduces leukocyte adherence and vascular injury in the newborn pig cerebral circulation after asphyxia. Editorial comment. Stroke 2000; 31: 2223.

11. Wisselink W, Patetsios P, Panetta TF, et al. Medium molecular weight pentastarch reduces reperfusion injury by decreasing capillary leak in an animal model of spinal cord ischemia. J Vasc Surg 1998; 27: 109-16.

12. Kaplan SS, Park TS, Gonzales ER, Gidday JM. Hydroxyethyl starch reduces leukocyte adherence and vascular injury in the newborn pig cerebral circulation after asphyxia. Stroke 2000; 31: 2218-23.

13. Rudolf J, HES in Acute Stroke Study Group. Hydroxyethyl starch for hypervolemic hemodilution in patients with acute ischemic stroke: a randomized, placebo-controlled phase II safety study. Cerebrovasc Dis 2002; 14: 33-41.

14. Woessner R, Grauer MT, Dieterich HJ, et al. Influence of a longterm, high-dose volume therapy with $6 \%$ hydroxyethyl starch $130 / 0.4$ or crystalloid solution on hemodynamics, rheology and hemostasis in patients with acute ischemic stroke. Results of a randomized, placebo-controlled, double-blind study. Pathophysiol Haemost Thromb 2003; 33: 121-6.

15. Xiong L, Lei C, Wang $Q, L i W$. Acute normovolaemic haemodilution with a novel hydroxyethyl starch (130/0.4) reduces focal cerebral ischaemic injury in rats. Eur J Anaesthesiol 2008; 25: 581-8.

16. Trieb J, Baron JF, Grauer MT, Strauss RG. An international view of hydroxyethyl starches. Intensive Care Med 1999; 25: 258-68.

17. Ruttmann TG, James MFM, Aronson I. In vivo investigation into the effects of haemodilution with hydroxyethyl starch (200/0.5) and normal saline on coagulation. Br J Anaesth 1998; 80: 612-6.

18. Navickis RJ, Haynes GR, Wilkes MM. Effect of hydroxyethyl starch on bleeding after cardiopulmonary bypass: a meta-analysis of randomized trials. J Thorac Cardiovasc Surg 2012; 144: 223-30.

19. Leuschner J, Opitz J, Winkler A, Scharpf R, Bepperling F. Tissue storage of 14C-labelled hydroxyethyl starch (HES) 130/0.4 and HES 200/0.5 after repeated intravenous administration to rats. Drugs R D 2003; 4: 331-8.

20. Neff TA, Doelberg M, Jungheinrich C, Sauerland A, Spahn DR, Stocker $R$. Repetitive large-dose infusion of the novel hydroxyethyl starch 130/0.4 in patients with severe head injury. Anesth Analg 2003; 96: 1453-9.

21. Haynes GR. Is hydroxyethyl starch safe in brain injury? Anesth Analg 2004; 99: 620. author reply 620-2.

22. Wiedermann CJ. Complications of hydroxyethyl starch in acute ischemic stroke and other brain injuries. Pathophysiol Haemost Thromb 2003; 33: 225-8.

23. Perner A, Haase N, Guttormsen AB, 6S Trial Group; Scandinavian Critical Care Trials Group, et al. Hydroxyethyl starch 130/0.42 versus Ringer's acetate in severe sepsis. N Engl J Med 2012; 367: 124-34.

24. Bayer O, Reinhart K, Kohl M, et al. Effects of fluid resuscitation with synthetic colloids or crystalloids alone on shock reversal, fluid balance, and patient outcomes in patients with severe sepsis: 
a prospective sequential analysis. Crit Care Med 2012; 40: 2543-51.

25. Myburgh JA, Finfer S, Bellomo R, Billot L, Cass A, Gattas D, Glass $P$, Lipman $J$, Liu B, McArthur $C$, McGuinness $S$, Rajbhandari D, Taylor CB, Webb SA; the CHEST Investigators and the Australian and New Zealand Intensive Care Society Clinical Trials Group. Hydroxyethyl starch or saline for fluid resuscitation in intensive care. N Engl J Med 2012; 367(20): 1901-11.

26. Wiedermann CJ. Hydroxyethyl starch-can the safety problems be ignored? Wien Klin Wochenschr 2004; 116: 583-94.

27. Perel P, Roberts I. Colloids versus crystalloids for fluid resuscitation in critically ill patients. Cochrane Database Syst Rev 2012; 6: CD000567. 